\title{
Ontological Assumptions of Psychiatric Taxonomy: Main Rival Positions and Their Critical Assessment
}

\author{
Panagiotis Oulis \\ Department of Psychiatry, Eginition Hospital, Athens University, Athens, Greece
}

\section{Key Words}

Psychiatric nosology • Psychopathology, classification • Psychopathology, taxonomy · Ontology $\cdot$ Idealism • Nominalism • Realism

\begin{abstract}
The scientific classification of human psychopathology raises a host of important philosophical issues ranging over all major areas of philosophical inquiry; logic, semantics, ontology, epistemology and ethics. The paper focuses on the major views of the ontological status of our psychodiagnostic constructs; idealism, nominalism and finally, neoaristotelian realism. Idealism holds that our psychodiagnostic constructs represent ideal essences, instantiated imperfectly by individual mental patients of the same psychodiagnostic class. These ideal essences are construed either as abstract forms dwelling in an autonomous realm of eternal abstract objects - platonistic idealism - or as ideal objects emerging in and grasped directly by a pure or absolute consciousness husserlian idealism. By contrast, nominalism claims that our psychodiagnostic constructs are nothing but conventional common names of sets of individual mental patients, invented and adopted on purely pragmatic grounds such as their practical utility, especially in predicting their clinical course and outcome under various types of treatment. Finally, neoaristotelian realism holds that, though our psychodiagnostic constructs are concepts, and thus of our own making, they
\end{abstract}

are not arbitrary ones but grounded in objective features shared by individual mental patients of a given psychodiagnostic class. It is argued that both idealism and nominalism are flawed and thus untenable - though for different reasons - and a refined version of neoaristotelian realism defended.

Copyright $\odot 2008$ S. Karger AG, Basel

\section{Introduction}

Ontological assumptions in the taxonomy of human psychopathology are philosophical hypotheses about the factual correlates, if any, of our classificatory constructs. In other words, they are tentative and programmatic answers to the question about what these constructs represent or what they stand for. In this short paper, I intend to provide both a brief explication of the main rival positions and their critical assessment. I will focus my analysis on the constructs belonging to the basic level of contemporary psychodiagnostic classification schemes, namely to constructs such as schizophrenic disorders, leaving aside both their superordinate and subordinate constructs, such as 'psychotic disorders' and 'paranoid schizophrenia', respectively. However, for illustrative purposes, I will also deal with constructs drawn from the conceptual corpus of general clinical psychopathology, such as those of auditory hallucinations or psychomotor

\section{KARGER \\ Fax +4161306 1234 E-Mail karger@karger.ch} www.karger.com

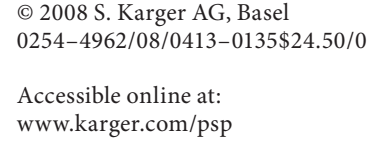

Panagiotis Oulis

Eginition Hospital, Department of Psychiatry

Athens University

GR-11528 Athens (Greece)

Tel. +30 210728 9408, Fax +30 210724 2020, E-Mail oulisp@med.uoa.gr 
agitation. An important critical review of the continental tradition in psychopathology recommends the careful distinction of clinical psychopathology as a provider of isolated symptoms with predominantly diagnostic and nosographic significance from general psychopathology as a promoter of meaningful connections holding among mental patients' pathological experiences [1]. In any event, the ontological hypotheses in question are closely related to the semantic assumptions or hypotheses which, in general, link conceptual items to factual ones providing thus factual semantic interpretations of the former in terms of the latter. However, in order to proceed to the factual semantic interpretation of our constructs, we first have to make definite ontological assumptions about the general kinds of existing factual items and their ways of being; otherwise, there will be no factual items for our constructs to be paired with. Therefore, ontology precedes semantics, not the other way round. Moreover, both precede epistemology, which deals with the justification of our already semantically interpreted knowledge - claims about factual items presumed to exist. Consequently, in the following I will leave aside crucial epistemological issues in the taxonomy of human psychopathology, such as the epistemological claim of the supposedly atheoretical character of latest DSM revisions [2-4]. However, the ontological assumptions, whose primacy I stressed previously, are epistemologically highly abstract and thus theoretical ones. Moreover, it is a solidly established fact by scientific psychology that even direct scientific observation is hypothesis driven. Both of these assertions imply the impossibility of presuppositionless scientific classification systems. Nevertheless, they are compatible with the weaker claim of the DSM to be agnostic with respect to the fine-grained causation of most categories of mental disorder included therein [24]. Finally, I will also leave aside here the analysis of the foundational concept of mental disorder [5], acknowledging, however, its multifaceted nature with at once ontological, semantic, epistemological and normative - especially moral and legal - components. Suffice it here to state, without further argument, that although its core meaning must be psychobiological - since only individual organisms of sufficient complexity are bearers of mental functions and thus susceptible to mental disorders - its applicability in psychiatric practice requires the enrichment of this core with clinical, diagnostic, moral and social layers of meaning as well.

We can distinguish at least three main ontological positions on the topic we are dealing with; idealism, nominalism and, finally, neoaristotelian realism.

\section{Idealism}

Idealism holds that our psychodiagnostic constructs stand for or represent ideal essences which are only imperfectly instantiated or exemplified in the individual mental patients, members of the same psychodiagnostic class. In the Platonist tradition, these ideal essences are conceived as abstract forms or archetypes dwelling in an autonomous realm of eternal ideal objects. By contrast, in the tradition of Edmund Husserl's philosophical phenomenology they are conceived as ideal objects emerging in phenomenologists' pure consciousness [6].

However, idealism is hardly consistent with the bulk of contemporary scientific knowledge, both natural and social, and has understandably very few supporters. More precisely, the ontological assumption derived from Platonism that our taxonomic constructs might represent autonomous ideal essences or eternal forms, as well as its companion epistemological thesis that they are discovered by psychopathologists through a special faculty of intuition capable to transcend the factual world and penetrate into the ideal world, seem clearly implausible. Likewise, the assumption derived from Husserl's idealism, according to which the essences of psychopathological disorders emerge as ideal objects in psychopathologists' pure consciousness, which grasps them directly and infallibly, seems also quite implausible. Nevertheless, Husserl's thesis, nominalistically reformulated via the methodological work of Max Weber in the social sciences and its systematic application to the study of human psychopathology by Karl Jaspers, remains very influential owing especially to the joint work of Michael Allan Schwartz and Osborne Wiggins. However, the issue of the influence of Husserl's philosophical phenomenology on Jaspers' methodological work in psychopathology remains still quite controversial [7-9]. Jaspers himself, at least implicitly, held that this influence was minimal [10, p. 47].

\section{Nominalism}

Nominalism, especially in its strong or radical version, denies the reality of both generic or universal and individual or particular properties of existing individual things. However, a nominalist variant known as trope theory, which I will not discuss further here, admits the existence of individual or particularized properties called 'tropes', claiming instead that individual things are nothing but bundles of such tropes. Strictly speaking, the ontology of radical nominalism admits only particular or 
individual things. Confronted with the real possibility of subsuming different individual mental patients to the same psychodiagnostic class and asked for an explanation, the radical nominalist typically claims that the overall similarity of its individual members constitutes a primitive not further analyzable or brute fact, not requiring thus for its explanation the admission of their possession of common or generic properties [see 11, pp. 59-60]. Radical nominalism in the taxonomy of human psychopathology amounts to the claim that our psychodiagnostic constructs are nothing but conventional common names of sets of individual mental patients, such as for example 'schizophrenia'. According to radical nominalism, these conventional common names are invented and accepted on purely pragmatic grounds such as their utility, especially their utility in collectively predicting patients' clinical course and outcome under various types of therapeutic intervention.

However, to begin with, individual patients' clinical psychopathology consists in the emergence of generic psychopathological properties and the sequences of their changes or processes, experiential or behavioral ones, such as for example verbal auditory hallucinations or psychomotor agitation, respectively. More precisely, external verbal auditory hallucinations of individual patients are individual experiential properties of theirs or rather processes unfolding episodically in their auditory consciousness. Nevertheless, despite their undeniable particularities in each individual case, all external verbal auditory hallucinations are strictly similar or equal in some crucial respects, such as being genuine experiences of patients' auditory consciousness without any external stimulation of their auditory sensory systems, being beyond or escaping their volitional control, referring essentially to major aspects of patients' life, etc. It is precisely these objective common aspects that ground individual patients' membership in the same psychopathological class, namely, in our example, the class of patients with external verbal auditory hallucinations. Furthermore, the same holds for all other major psychopathological disturbances of human mental functions; obsessions, compulsions, delusions, formal thought disorders, depersonalization, etc. are nothing but such generic psychopathological properties, though of course immanent to the psychopathological experiences or behaviors of individual mental patients, in sharp contrast to both their conception as enjoying an autonomous existence according to Platonism, as well as their construal as ideal contents of psychopathologists' pure or absolute consciousness according to Husserlianism.

Ontological Assumptions of Psychiatric Taxonomy
Besides, individual patients' overall clinical course and outcome is determined by the temporal course of such psychopathological generic properties and, thus, constitutes still another, though global, generic property of theirs. In addition, individual patients' types of response to various individual treatments constitute further generic properties of theirs, though relational ones, since they are possessed by the pairs patients/treatments. Moreover, strict nominalism, by denying the reality of generic psychopathological properties and processes, leaves the practical utility or predictive validity of our psychodiagnostic constructs totally unexplained. In sum, radical nominalism is both untenable and explanatorily deeply deficient. Worse still, radical nominalism is also often self-refuting. For example, the great British clinical psychiatrist, late Bob Kendell, at least in several passages of his classic The Role of Diagnosis in Psychiatry [12, pp. 21-23], tried to explicitly defend such a radical nominalist position by claiming that the sole justification of our concept of schizophrenia derives from its 'utility'. More precisely, Kendell thought that disease Platonism and strict nominalism exhausted all possible ontological options in the field of disease taxonomy. Thus, correctly rejecting Platonism, he opted for nominalism, claiming tellingly that 'diseases, tuberculosis, as well as schizophrenia, are nothing but man-made abstractions, inventions justified only for their convenience and liable at any time to be adjusted or discarded' [12, p. 21]. Nevertheless, in the same work, Kendell conceded that the utility of the concept of schizophrenia is grounded jointly in

(a) the universality of the behavioral and experiential clinical features of individual schizophrenic patients,

(b) the universality of their diminished reproductive fitness,

(c) the genetic/hereditary nature, at least in part, of patients' predisposition or vulnerability to the disorder, and

(d) schizophrenic patients' therapeutic response to antipsychotic medications.

However, all the preceding four features or clusters of features are nothing but generic properties or generic property clusters, whether dispositional or manifest, intrinsic or relational ones, shared by most individual schizophrenic patients. Moreover, all these generic properties or clusters of properties are admittedly lawfully interrelated, these lawful patterns being further, complex, generic properties of individual schizophrenic patients. Thus, the psychodiagnostic construct of schizophrenia represents after all a cluster of lawfully interrelated generic properties, which, jointly, objectively ground patients' common membership to the same psychodiagnostic or taxonomic class.

Psychopathology 2008;41:135-140 
Likewise, Wiggins and Schwartz, in their joint paper titled 'The limits of psychiatric knowledge and the problem of classification', tried to defend the claim that our psychodiagnostic constructs are more or less arbitrary simple names, in contrast to the 'naturalistic' concepts of natural sciences, since they fail to 'carve up' human psychopathology to its 'joints' [13, pp. 94-97]. Nevertheless, in the same study, Wiggins and Schwartz admitted that the diagnostic criteria of each psychodiagnostic construct sketch descriptive models of patients' clinical psychopathology, though highly idealized and simplified ones, and that consequently, they are revisable in the light of novel psychopathological research findings relevant to them. However, their joint requirements of descriptive adequacy and essential revisability in order to better fit novel scientific research findings imply that our psychodiagnostic constructs, far from being more or less arbitrary names, represent after all, though crudely and at best approximately, patients' common or generic, mostly clinical, psychopathological properties and their lawful interrelations, a result hardly compatible with their selfprofessed nominalistic antirealism.

\section{Neoaristotelian Realism}

Finally, neoaristotelian realism holds that although our psychodiagnostic constructs are concepts and thus of our own making, they are neither arbitrary nor conventional ones, since they are grounded in common clusters of objective features shared by individual mental patients of the same psychodiagnostic class. In other words, they are grounded in patients' possession of common or generic psychopathological properties and their lawful interrelations. One important consequence of this thesis is that the problem of whether psychiatric taxonomies classify people or disorders is ontologically spurious, since there cannot exist disorders detached from people having them. In short, psychiatric classifications cannot but classify mentally disordered people or people with mental disorders.

However, we should distinguish, though not detach, the clusters of generic psychopathological surface properties and processes represented by our clinical diagnostic or identifying criteria from the clusters of yet largely unknown deep, source or root properties and processes, crucial aspects of their pathogenetic and above all pathophysiological mechanisms, which most importantly individuate psychopathological kinds [for a similar distinction in the field of biological taxonomy see 14, chapt. 7]. Obviously, unlike biological species, psychopathological kinds cannot be kinds of individuals but only kinds of properties of individuals and processes in individuals. In other words, like physical diseases, the mental ones are not kinds of substances but kinds of organismic processes, namely of mental - experiential and behavioral - ones. Regrettably, the currently prevailing classification systems such as DSM-IV are mainly grounded only in behavioral features and patterns. It goes without saying that deeper psychological and neurobiological ones are vitally needed in order to ground their shaky validity. However, this in turn requires the as yet very difficult to achieve ontological conciliation of human and natural sciences as well as their methodological integration [15]. In addition, social factors and patterns also have to be taken into account in order to better understand the often strong social component of the causation of mental disorders [16, 17]. Moreover, strictly speaking, each psychopathological kind is fully individuated by both clusters of properties and processes, namely the surface and the deep ones. Therefore, the clusters of characteristic clinical features represented by the diagnostic criteria of most contemporary categories of mental disorder individuate only syndromic psychopathological or taxonomic kinds. By contrast, both clusters of characteristic clinical features and characteristic, yet largely unknown, underlying mechanisms individuate genuine psychopathological or nosological kinds.

Furthermore, according to ontological realism in general, we should carefully distinguish the ontic level, focus of the present paper, from the epistemic level. Psychopathological properties and psychopathological law patterns belong to the former, whereas diagnostic criteria, clinical law-like generalizations and pathogenetic and pathophysiological hypotheses belong to the latter.

Nevertheless, we should acknowledge that many philosophical and scientific problems stand on our epistemic way from taxonomic to nosological psychopathological kinds. One such major obstacle to the discovery of psychopathological lawful patterns, even merely clinical ones, stems from the current excessive proliferation of disjunctive and negative diagnostic criteria, both of which in addition contribute to the artificial inflation of psychiatric comorbidity. Disjunctive diagnostic criteria, in the inclusive sense of the logical operation of disjunction, i.e. and/or, predominate in the diagnostic inclusion criteria, whereas negative diagnostic criteria abound in the exclusion ones. However, since there are neither disjunctive nor negative generic properties of things in general, these criteria fail to represent any genuine psychopathological properties. For example, 'grossly disorganized or cataton- 
ic behavior', a subcriterion of diagnostic criterion A for schizophrenic disorders [see 6, p. 285], though respectable as a disjunctive predicate, fails to represent any genuine disjunctive property; patients may exhibit grossly disorganized behavior, a genuine behavioral property of theirs, or they may exhibit catatonic behavior, another genuine behavioral property of theirs, or finally, they may exhibit the conjunction of the preceding two genuine properties, namely both grossly disorganized and catatonic behavior. However, there is no disjunctive property of theirs representable by the disjunctive predicate of our example. Indeed, if such a disjunctive property existed, then patients exhibiting only disorganized behavior and patients exhibiting only catatonic behavior should be equal or identical with respect to this 'property'. Likewise, 'not having schizoaffective disorder', part of diagnostic criterion $D$ for schizophrenic disorders [6, p. 285], though respectable as a negative predicate, fails to represent any genuine supposedly negative property; not possessing a positive property does not amount to possessing a negative one. In our example, there is no property consisting in not having a schizoaffective disorder. Any two mental patients with no schizoaffective disorder need not have any common psychodiagnostic property; for example the first one may have a schizophrenic disorder and the second one an affective disorder, though both satisfy the same above-mentioned negative predicate [for similar and additional arguments against the admissibility of disjunctive and negative properties in ontology, see 18 , pp. 82-83]. In this respect, the expression 'negative symptoms' - widely used with respect to the schizophrenic disorders - is philosophically ill-formed and thus misleading.

Consequently, the psychopathological classes formed through the clinical application of such criteria fail to possess common or universally shared clinical psychopathological features. Furthermore, since lawful patterns in general interrelate generic properties of individuals, psychodiagnostic classes of current psychiatric classification schemes fail to exhibit such patterns as well. In order to remedy the resulting excessive clinical heterogeneity exhibited by mental patients belonging to the same psychodiagnostic class, more restrictive or conjunctive in the logical sense diagnostic criteria are obviously needed. Since such criteria will also considerably reduce the diagnostic coverage of our official classification systems, their application must be reserved for research purposes, at least initially.

At this point, a brief and passing remark about the socalled prototype approach to psychiatric classification is in order [19]. This approach is quite appropriate for the initial acquisition in our clinical training and the subsequent routine application in our clinical practice of our psychodiagnostic constructs. However, it is totally inappropriate for the scientific advancement of psychopathological taxonomy or even detrimental to it, since it extols and consecrates clinical heterogeneity of each psychodiagnostic class to the highest possible degree. Finally, another brief remark on the dimensional approach to the classification of human psychopathology is also in order $[20,21]$. Dimensions and categories are neither incommensurable nor mutually exclusive but, on the contrary, quite compatible and even complementary. In general, the vast majority of both single psychopathological features and their syndromic clusters come in various degrees of severity, and conversely, each degree of severity is necessarily the degree of severity of some clinical feature or syndrome or another.

However, the final choice of the order of priority in each diagnostic axis between categories and dimensions, that is, whether categories come first and are then graded according to levels of severity or dimensions come first and then categories are defined as definite ranges of variation along these dimensions, will ultimately be dictated by the form of the relevant - reasonably confirmed - psychopathological law statements.

\section{Conclusions}

I briefly reviewed three main positions on the ontological status of current psychiatric taxonomy: idealism, nominalism and finally, neoaristotelian realism. I found the first two ontological positions wanting and argued for the superiority of neoaristotelian realism. However, the defensibility of neoaristotelian realism requires the distinction - though not separation - between mere taxonomic psychopathological kinds and genuine nosological ones. I also argued that - at least in present-day psychiatry - psychopathological kinds are only taxonomic, not nosological ones, and pointed out some major philosophical and scientific obstacles - especially the excessive proliferation of disjunctive and negative diagnostic criteria - seriously hindering further scientific progress in the classification of human psychopathology.

\section{Acknowledgments}

The author wishes to thank Stathis Psillos, $\mathrm{PhD}$, for his insightful comments on a previous draft and George Konstantanopoulos for precious help in manuscript preparation. 


\section{References}

1 Rossi Monti M, Stanghellini G: Psychopathology: an edgeless razor? Compr Psychiatry 1996;37:196-204.

2 American Psychiatric Association: Diagnostic and Statistical Manual of Mental Disorders, ed 3. Washington, American Psychiatric Association, 1980.

3 American Psychiatric Association: Diagnostic and Statistical Manual of Mental Disorders, ed 3, rev. Washington, American Psychiatric Association, 1987.

4 American Psychiatric Association: Diagnostic and Statistical Manual, ed 4, Washington, American Psychiatric Association, 1994.

5 Berganza CE, Mezzich JE, Pouncey C: Concepts of disease: their relevance for psychiatric diagnosis. Psychopathology 2005;38: 166-170.

6 Husserl E: Phänomenologie, Encyclopaedia Britannica Artikel (1927); in Husserl E: Die phänomenologische Methode. Ausgewählte Texte (ed Held K). Stuttgart, Reclam, 1998, vol 1, pp 198-206.

7 Berrios GE: Phenomenology, psychopathology and Jaspers: was there ever a relationship? Compr Psychiatry 1993;34:213-220.
8 Langenbach M: Phenomenology, intentionality and mental experiences: Edmund Husserl's Logische Untersuchungen and the first edition of Karl Jaspers' Allgemeine Psychopathologie. Hist Psychiatry 1995;6:209224.

9 Wiggins OP, Schwartz MA: Edmund Husserl's influence on Karl Jaspers's phenomenology. Philos Psychiatr Psychol 1997;2:247266.

10 Jaspers K: Allgemeine Psychopathologie, ed 5. Berlin, Springer, 1948.

11 Loux MJ: Metaphysics. A Contemporary Introduction. London, Routledge, 1998.

12 Kendell RE: The Role of Diagnosis in Psychiatry. Oxford, Blackwell, 1975.

13 Wiggins OP, Schwartz MA: The limits of psychiatric knowledge and the problem of classification; in Sadler JZ, Wiggins OP, Schwartz MA (eds): Philosophical Perspectives on Psychiatric Diagnostic Classification. Baltimore, Johns Hopkins University Press, 1994, pp 89-103.

14 Mahner M, Bunge M: Foundations of Biophilosophy. Berlin, Springer 1997.
15 Mundt C: Psychopathologie heute; in Kisker KP, Lauter H, Meyer JE, Mueller C, Stroemgren E (eds): Brennpunkte der Psychiatrie. Berlin, Springer, 1989, pp 147-184.

16 Dohrenwend BP (ed): Adversity, Stress and Psychopathology. New York, Oxford University Press, 1998.

17 Berkmann LF, Kawachi I (eds): Social Epidemiology. New York, Oxford University Press, 2000.

18 Armstrong DM: Universals. An Opinionated Introduction. Boulder, Westview Press, 1989.

19 Jablensky A: Categories, dimensions and prototypes: critical issues for psychiatric classification. Psychopathology 2005;38: 201-205.

20 Widiger T: A dimensional model of psychopathology. Psychopathology 2005;38:211214.

21 Mackinejad K, Sharifi V: Wittgenstein's philosophy and a dimensional approach to the classification of mental disorders: a preliminary scheme. Psychopathology 2006;39: 126-129. 